\title{
Mixed-lineage kinase 3 pharmacological inhibition attenuates murine nonalcoholic steatohepatitis
}

\author{
Kyoko Tomita, ${ }^{1}$ Rohit Kohli, ${ }^{2}$ Brittany L. MacLaurin, ${ }^{1}$ Petra Hirsova,, Qianqian Guo, ${ }^{1}$ \\ Luz H. Gutierrez Sanchez, ${ }^{3}$ Harris A. Gelbard, ${ }^{4}$ Burns C. Blaxall, ${ }^{5}$ and Samar H. Ibrahim ${ }^{1,3}$ \\ 'Division of Gastroenterology and Hepatology, Mayo Clinic, Rochester, Minnesota, USA. Division of Pediatric \\ Gastroenterology, Children's Hospital Los Angeles, Los Angeles, California, USA. ${ }^{3}$ Division of Pediatric Gastroenterology, \\ Mayo Clinic, Rochester, Minnesota, USA. ${ }^{4}$ Center for Neurotherapeutics Discovery and Department of Neurology, \\ University of Rochester Medical Center, Rochester, New York, USA. ${ }^{5}$ Department of Pediatrics, University of Cincinnati, \\ Cincinnati Ohio, USA.
}

With the increase in obesity worldwide, its associated comorbidities, including nonalcoholic steatohepatitis (NASH), have become a public health problem that still lacks effective therapy. We have previously reported that mixed-lineage kinase 3-deficient (MLK3-deficient) mice are protected against diet-induced NASH. Given the critical need to identify new therapeutic agents, we sought to examine whether the small-molecule MLK3 inhibitor URMCO99 would be effective in reversing diet-induced murine NASH. C57BL/6] mice were fed either a diet high in saturated fat, fructose, and cholesterol (FFC), or a chow diet for 24 weeks. Mice were treated with either URMC099 (10 $\mathrm{mg} / \mathrm{kg}$ ) twice daily by intraperitoneal injection or its vehicle during the last 2 weeks of the feeding study. FFC-fed mice receiving URMCO9g had similar body weight, caloric intake, homeostatic model assessment of insulin resistance, metabolic phenotype, and hepatic steatosis compared with vehicle-treated mice. Furthermore, FFC-fed mice treated with URMCO9g had less hepatic macrophage infiltration, activation, and proinflammatory polarization, as well as less liver injury and fibrosis when compared with vehicle-treated mice. In conclusion, URMCO9g is well tolerated in mice without obvious toxicities and appears to be efficacious in reversing diet-induced NASH. Hence, URMCO99 may serve as a therapeutic agent in human NASH.

Conflict of interest: The authors have declared that no conflict of interest exists.

Submitted: April 7, 2017 Accepted: June 29, 2017 Published: August 3, 2017

\section{Reference information:} JCI Insight. 2017;2(15):e94488. https://doi.org/10.1172/jci. insight.94488.

\section{Introduction}

With the global epidemic of obesity, nonalcoholic fatty liver disease (NAFLD) disease and its associated comorbidities including insulin resistance and cardiovascular disease have emerged as a significant public health problem (1). Approximately $44 \%$ of patients with NAFLD progress to nonalcoholic steatohepatitis (NASH) (2), the more severe phenotype of the disease characterized by hepatic inflammation and fibrosis in addition to steatosis. Patients with NASH fibrosis are at high risk for rapid progression to end-stage liver disease (3). Currently, NASH is the second leading cause of liver transplantation among adults on the waiting list (4). Although NASH treatment is limited to weight reduction, given the large number of patients that are unable to achieve and maintain weight loss, there is a critical, unmet need for effective pharmacological therapy with antiinflammatory and antifibrotic properties.

Excess circulating saturated free fatty acids (SFAs) in patients with NASH (5) culminates in hepatocyte lipoapoptosis $(6,7)$, a pathologic hallmark of NASH that correlates with disease severity $(8,9)$. Mixedlineage kinase type 3 (MLK3) is a ubiquitously expressed serine/threonine mitogen-activated protein kinase kinase kinase (MAPKKK) that functions in a phosphorylation relay module to control the activity of downstream MAPKs $(10,11)$, and mediates SFA-induced hepatocyte lipoapoptosis $(12,13)$. Likewise, the role of MLK3 as a proapoptotic kinase has been established in different models of cell injury (14-16).

In addition to hepatocyte lipoapoptosis, hepatic lipotoxicity is associated with inflammatory infiltrates, which further promote liver injury and fibrosis $(17,18)$. Trafficking of inflammatory monocytes from the blood and activation of the resident macrophages within the liver are essential pathogenic mechanisms in the progression of NAFLD (17). At the early stage of NASH, blood monocytes, recruited into the liver in 
response to the liver microenvironment, undergo proinflammatory polarization (19), resulting in cytotoxic and inflammatory activation (20). Proinflammatory monocytes promote hepatic stellate cell (HSC) activation and transforming growth factor $\beta$ release, leading to the progression of liver fibrosis (21).

We have reported previously that $M L K 3^{-/-}$mice are protected against liver injury, fibrosis, and inflammation in a murine model of NASH-inducing diet $(22,23)$. We also demonstrated that hepatocytes under lipotoxic stress release chemotactic extracellular vesicles (EVs) by an MLK3-dependent mechanism (23); these vesicles are enriched with the potent macrophage chemotactic ligand (C-X-C motif) ligand 10 (CXCL10), expression of which is regulated by MLK3 in hepatocytes (11). Furthermore, mice fed the $\mathrm{NASH}$-inducing diet have increased CXCL10 in plasma EVs in an MLK3-dependent fashion (23), and CXCL10 $10^{-1}$ mice are protected against diet-induced NASH (24). Likewise, MLK3 proinflammatory properties have been recently recognized in different disease models (25-27).

Bolstered by our data supporting MLK3 as a crucial proinflammatory and proapoptotic mediator in our preclinical in vitro and in vivo model of $\operatorname{NASH}(22,23)$, we elected to test the protective effect of the MLK3 pharmacological inhibitor URMC099 in our murine model of diet-induced NASH and obesity. URMC099 is a selective small-molecule MLK3 pharmacological inhibitor that was developed for human use. URMC099 has nanomolar potency for MLK3 inhibition and a favorable metabolic and toxicity profile (25). Herein, we demonstrate a reduction in liver inflammation, liver injury, and fibrosis in URMC099. treated mice, independent of effects on weight gain and parameters of the metabolic syndrome. We also demonstrate a direct antiinflammatory role of URMC099 through the attenuation of macrophage activation under lipotoxic conditions in vitro.

\section{Results}

The metabolic phenotype of URMC099-treated FFC-fed mice. A diet high in saturated fat, fructose, and cholesterol (FFC) induced a significant increase in body weight compared with the standard chow over the 24-week study period in both URMC099-treated and vehicle-treated mice (Figure 1A). Total body weights were similar between URMC099-treated and vehicle-treated mice on standard chow or the FFC diet (Figure 1A). The FFC diet-induced increase in body weight is primarily due to an increase in fat mass, as we previously reported (24). Caloric intake was similar between the FFC-fed mice during the treatment period with either URMC099 or vehicle (Figure 1B). No differences in body composition (percentage of lean mass versus fat mass) were observed between the FFC-fed mice treated with either URMC099 or vehicle (Figure 1C). Furthermore, energy expenditure (Figure 1D), physical activity (Figure 1E), and respiratory quotient (Figure 1F) were similar between FFC-fed URMC099-treated versus vehicle-treated mice. Likewise, homeostasis model assessment of insulin resistance (HOMA-IR) (Figure 1G) was similar between the 2 treatment groups on FFC diet, suggesting that URMC099 does not affect insulin response. Of note, the HOMA-IR in the current study was lower than what we previously reported (24), which we attributed to the weight loss that mice experienced over the last 2 weeks of the study period, secondary to the twiceper-day intraperitoneal (i.p.) injections and the stress caused by the Comprehensive Lab Animal Monitoring System (CLAMS) study (Figure 1H); the weight loss was similar between the groups on the same diet regardless of the treatment condition. Thus, overall FFC-fed mice tolerated URMC099 well; mice were active during the study period without noted adverse effects or significant weight loss when compared with vehicle-treated mice on the same diet. URMC099 treatment did not alter the metabolic phenotype induced by the FFC diet in our murine NASH model.

URMC099 treatment in mice reduces FFC-induced activation of MLK3 downstream targets in the liver. To show that URMC099 treatment exerts a direct inhibition of MLK3 downstream targets, we demonstrate significant reduction of the FFC diet-induced phosphorylation of the mitogen-activated protein kinase kinases (MAP2Ks) MKK3/MKK6, and the mitogen-activated protein kinase (MAPK) p38 (Figure 2A), as well as significant reduction of the mRNA expression of CXCL10 (Figure 2B) in liver homogenates of the FFC-fed, URMC099-treated mice compared with vehicle-treated mice on the same diet. These findings are consistent with our previous in vitro report that hepatocyte lipotoxicity triggers the activation of a MAPK signaling cascade consisting of the MAP3K MLK3, which activates the MAP2Ks MMK3/MKK6, and its downstream MAPK p38, resulting in the subsequent induction of the chemokine CXCL10. Likewise, URMC099 treatment in vitro inhibits this signaling cascade in hepatocytes under lipotoxic stress $(11,23)$.

URMC099 treatment does not reduce FFC diet-induced hepatic steatosis in mice. Histological examination of the liver by hematoxylin and eosin (H\&E) stain displayed a similar extent of steatosis in the FFC-fed mice treated 



$\mathbf{F}$



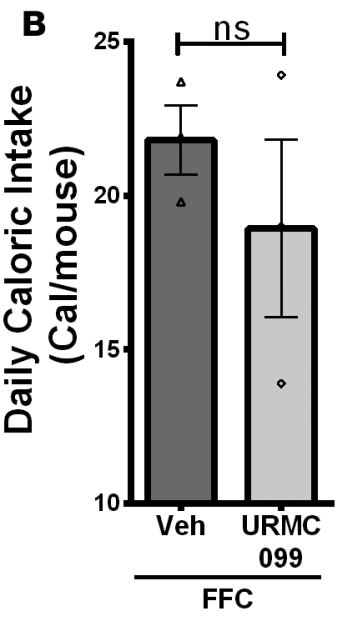

C

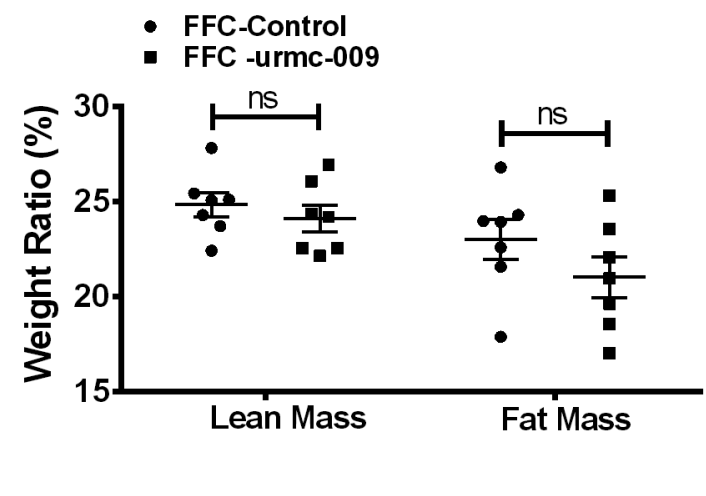

$\mathbf{E}$

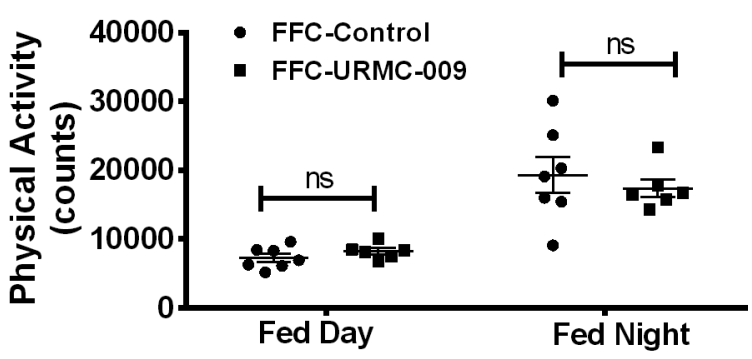

H

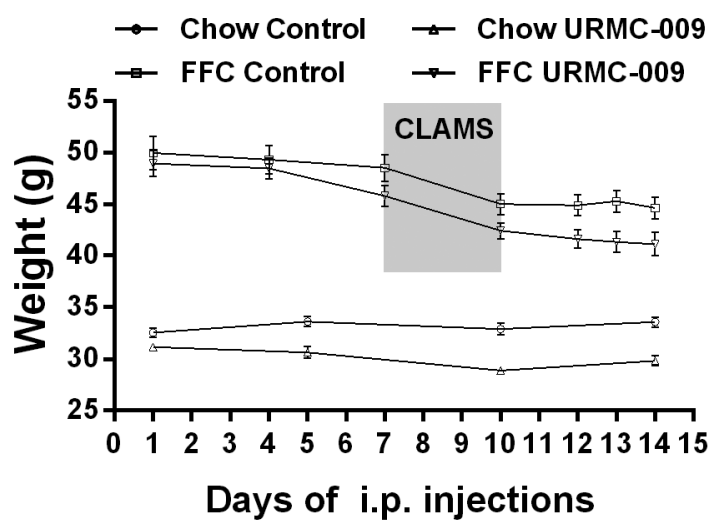

Figure 1. Metabolic phenotype of URMC099-treated, FFC-fed mice. WT [57BL/6) mice fed either chow or a diet high in saturated fat, fructose, and cholesterol (FFC) for 24 weeks were randomized to receive either URMC099 or vehicle i.p. twice daily during the last 2 weeks $(n=5-7)$. (A) Body weight at the time of sacrifice $(n=5-7)$. (B) Daily caloric intake (calories per day per mouse) during the period of i.p. injection $(n=3)$. (C) Weight ratio of lean mass (\%) and fat mass (\%) ( $(n=7)$. (D) Energy expenditure (kcal/hour $/ \mathrm{kg})(n=7)$. (E) Physical activity (counts) $(n=7)$. (F) Respiratory quotient $(n=7)$. (G) Homeostasis model assessment of insulin resistance (HOMA-IR) calculated from fasting glucose and insulin levels $(n=4-7)$. (H) Weight loss during the period of i.p. injections $(n=5-7)$. Data represent mean \pm SEM. Differences between the groups were compared using 1 -way ANOVA followed by Bonferroni's multiple comparisons test. ${ }^{* *} P<0.001$. ns, non-significant.

with URMC099 versus vehicle (Figure 3A). Likewise, steatosis assessment by coherent anti-Stokes Raman scattering (CARS) microscopy showed similar neutral triglyceride (TG) accumulation in the hepatocytes of the FFC-fed, URMC099- and vehicle-treated mice (Figure 3B). This observation was further confirmed biochemically by assessing the TG content in liver tissue, which was similar in FFC-fed, URMC099- and vehicle-treated mice (Figure 3C). These findings were consistent with the similar liver/body weight ratio in the 


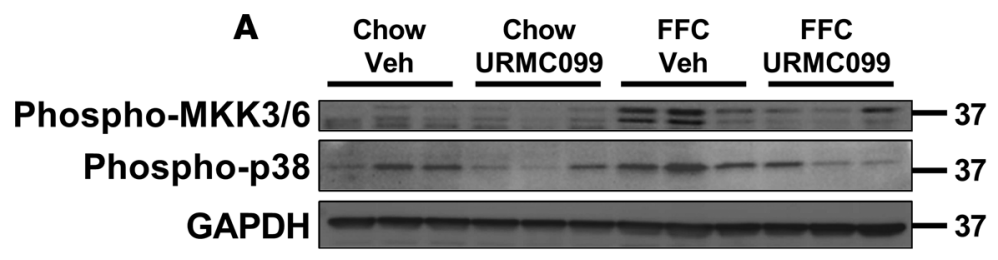

B

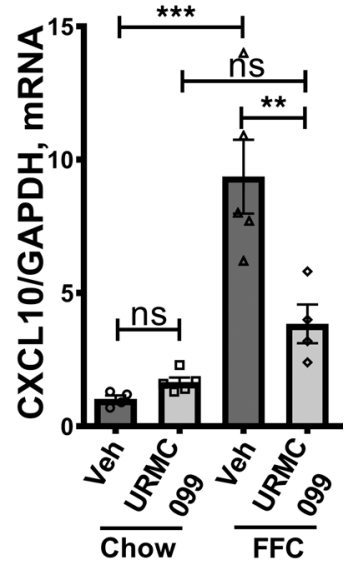





Figure 2. URMC099 in mice reduces FFC-induced activation of MLK3 downstream targets in the liver. WT C57BL/6] mice fed either chow or a diet high in saturated fat, fructose, and cholesterol (FFC) for 24 weeks were randomized to receive either URMCO99 or vehicle i.p. twice daily during the last 2 weeks. (A) Immunoblot was used to assess phospho-MKK3/MKK6, phospho-p38, and GAPDH protein levels in whole mouse liver lysates from the different experimental groups. Quantification of protein levels $(n=3)$ was performed with Image). (B) The mRNA expression of CXCL10 was assessed by real-time PCR $(n=4-5)$. Fold change was determined after normalization to GAPDH expression in liver tissue, and expressed relative to that observed in chow-fed, vehicle-treated mice. Data represent mean \pm standard error of the mean. Differences between the groups were compared using 1-way ANOVA followed by Bonferroni's multiple comparisons test. ${ }^{*} P<0.05,{ }^{*} P<0.01$, ${ }^{* * *} P<0.001$. ns, non-significant.

FFC-fed, URMC099- and vehicle-treated mice (Figure 3D). Although there were prominent inflammatory infiltrates in the FFC-fed, vehicle-treated mice, these infiltrates were reduced in the URMC099-treated mice on the same diet (Figure 3A). Thus, FFC-fed, URMC099-treated mice had hepatic steatosis similar to that of vehicle-treated mice on the same obesity-inducing diet, but display reduced hepatic inflammatory infiltrates.

URMC099 treatment protects mice against FFC diet-induced macrophage-associated hepatic inflammation. Based on our previous observation that MLK3 activation in hepatocytes under lipotoxic conditions induces the upregulation of the potent macrophage chemotactic ligand CXCL10 $(11,23)$, we first assessed the expression of CXCL10 in whole liver by quantitative real-time PCR to assess whether systemic administration of URMC099 suppressed CXCL10 induction in the liver. Interestingly, URMC099 treatment significantly reduced CXCL10 hepatic mRNA expression in FFC-fed mice (Figure 2A). Likewise, CXCL10 serum level was reduced in FFC-fed, URMC099-treated mice when compared with vehicle-treated mice on the same diet (Figure 4A). We then assessed whether the hepatic downregulation of CXCL10 is associated with reduced macrophage hepatic infiltration, a key element in the sterile inflammatory response in murine NASH (24). We examined hepatic macrophage infiltration by immunohistochemistry for macrophage galactose-specific lectin (Mac-2), a marker of macrophages engaged in phagocytosis (28). The FFC diet caused an increase in the Mac-2-immunopositive surface area, which was significantly reduced with URMC099 treatment but not with vehicle treatment (Figure 4B); this effect is consistent with the influence of URMC099 on macrophages with a pathologic phenotype in a different disease model (5). Likewise, vehicle-treated, FFC-fed mice had a significant increase in the hepatic mRNA expression of the general macrophage and monocyte markers cluster of differentiation (CD) 14, CD68, and F4/80 (Figure 4C and ref. 29), which were reduced in the FFC-fed, URMC099-treated mice. In addition, mRNA expression of IL-12p40, a known proinflammatory (M1) marker (30), was increased in the vehicle-treated, FFC-fed mice, and significantly reduced with URMC099 treatment in the mice on the same diet (Figure 4D). Moreover, the expression of blood monocyte-derived macrophage markers, including the inflammatory monocyte surface lymphocyte antigen 6 complex (Ly6C), and C-C chemokine receptor type 2 (CCR2) (31), were significantly augmented with the FFC diet, and reduced with URMC099 treatment (Figure 4E). Furthermore, mRNA expression of 
A

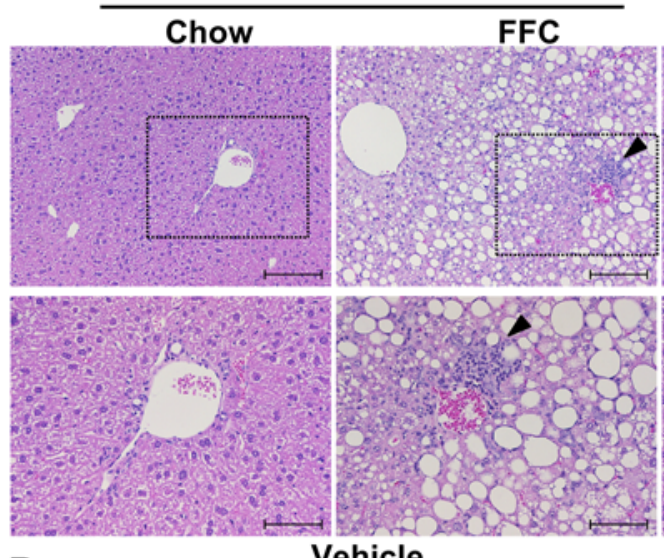

Vehicle

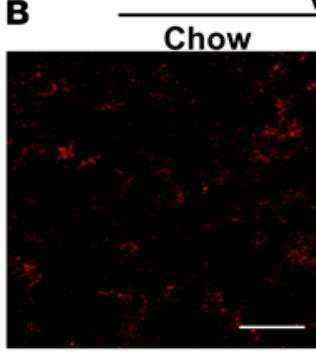

C



FFC



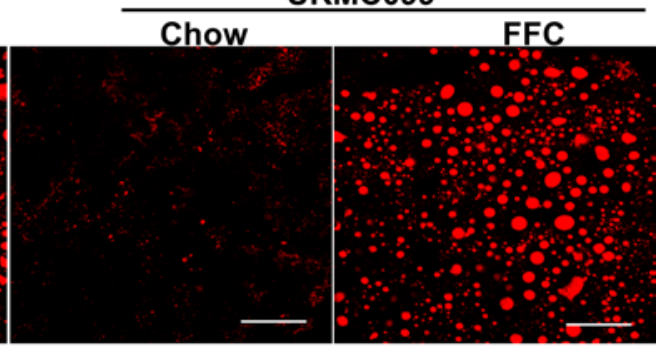

URMC099

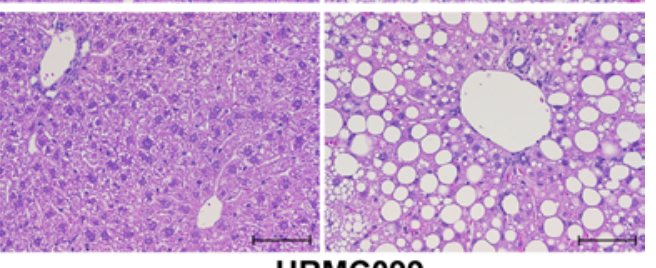

Chow

D

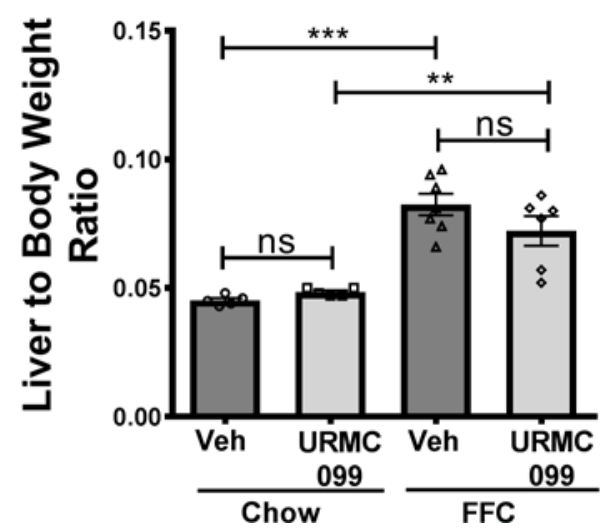

Figure 3. URMC099 treatment does not reduce the FFC diet-induced hepatic steatosis in mice. WT C57BL/6) mice were fed either chow or a diet high in saturated fat, fructose, and cholesterol (FFC) for 24 weeks, and URMCog9 or vehicle (Veh) was given twice daily i.p. during the last 2 weeks. (A) Fixed liver tissues were stained with hematoxylin and eosin (H\&E); black arrowheads indicate inflammatory infiltrates. Bottom row contains images enlarged from the boxed area in the corresponding panel in the top row. Scale bars: $100 \mu \mathrm{m}$ (top panel) and $50 \mu \mathrm{m}$ (bottom panel). (B) Label-free frozen liver tissue sections were imaged by coherent anti-Stokes Raman scattering (CARS) microscopy to visualize lipid droplets using a $\times 25$ objective. Scale bars: 50 $\mu \mathrm{m}$. (C) Neutral triglyceride content (mg/dl) in the liver was measured using a photometric absorbance-based technique ( $n=5-7)$. (D) Liver/body weight ratio was calculated $(n=5-7)$. Data represent mean \pm SEM. Differences between the groups were compared using 1-way ANOVA followed by Bonferroni's multiple comparisons test. ${ }^{* *} P<0.01,{ }^{* *} P<0.001$. ns, non-significant.

the macrophage cytokine and chemokine activation markers tumor necrosis factor tumor necrosis factor- $\alpha$ (TNF- $\alpha$ ) and monocyte chemotactic protein-1 (MCP-1) were increased in the FFC-fed, vehicle-treated mice, but significantly reduced with URMC099 treatment in the mice on the same diet (Figure 4F). These data are consistent with the reduced markers of macrophage hepatic accumulation and activation observed in $\mathrm{MLK}^{---}$mice on an obesity-inducing diet (refs. 22, 23, and Supplemental Table 1; supplemental material available online with this article; https://doi.org/10.1172/jci.insight.94488DS1). Taken together, these data demonstrate an aggregate reduction of proinflammatory macrophage hepatic infiltration, macrophage activation, and proinflammatory polarization in the FFC-fed, URMC099-treated mice.

FFC-fed, URMC099-treated mice are protected against FFC diet-induced liver injury and fibrosis. URMC099treated mice on the FFC diet had significantly lower serum alanine aminotransferase (ALT), alkaline phos- 

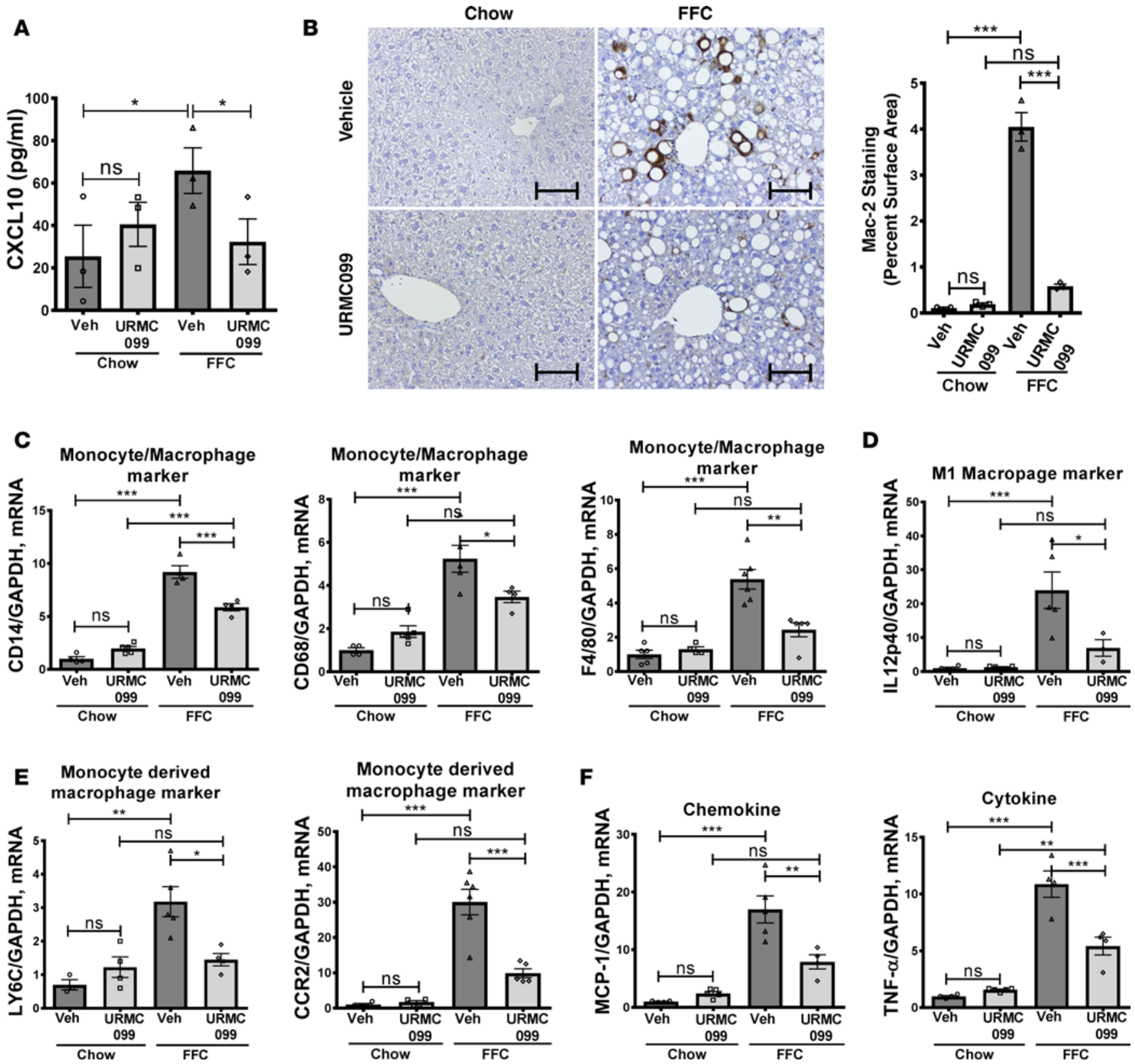

Figure 4. URMC099-treated mice have reduced FFC diet-induced macrophage-associated liver inflammation. WT (57BL/6) mice were fed either chow or a diet high in saturated fat, fructose, and cholesterol (FFC) for 24 weeks, and URMcog9 or vehicle (Veh) was given during the last 2 weeks. (A) CXCL10 levels in the serum were assessed by ELISA $(n=3)$. (B) Macrophage infiltration was evaluated by immunohistochemistry for macrophage galactose-specific lectin (Mac-2) antigen; Mac-2 staining was quantified in 10 random $\times 20$ microscopic fields and averaged for each animal ( $n=3$ ) by morphometry using Image software. Scale bars: $50 \mu \mathrm{m}$. Total RNA was extracted from liver tissue. The mRNA expression of (C) general monocyte and macrophage markers CD14, CD68, and F4/80 ( $n=4-6)$, (D) M1 macrophage marker IL-12p40 ( $n=4-5)$, (E) markers associated with monocyte-derived infiltrating macrophages CCR2 and LY6C $(n=4-5)$, and (F) the chemokine MCP-1 and the cytokine TNF- $\alpha$ were assessed by real-time PCR ( $n=4-5)$. Fold change was determined after normalization to CAPDH expression in liver tissue, and expressed relative to that observed in chow-fed, vehicletreated mice. Data represent mean \pm SEM. Differences between the groups were compared using 1-way ANOVA followed by Bonferroni's multiple comparisons test. ${ }^{*} P<0.05,{ }^{* *} P<0.01,{ }^{* *} P<0.001$. ns, non-significant.

phatase, and bile acid levels when compared with vehicle-treated mice on the same diet, consistent with reduced liver injury and cholestasis (Figure 5A). Likewise, FFC-fed, URMC099-treated mice were relatively protected against FFC diet-induced lipoapoptosis, as demonstrated by a significant reduction in the number of terminal deoxynucleotidyl transferase-mediated deoxyuridine triphosphate nick-end labelingpositive (TUNEL-positive) cells in the URMC099-treated mice versus vehicle-treated mice (Figure 5B). The protective effect of URMC099 against toxic lipid-induced hepatocyte apoptosis was further confirmed 


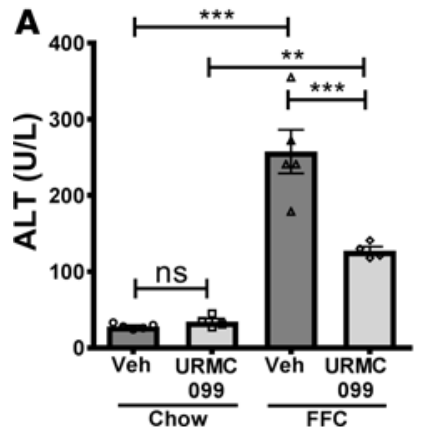

B

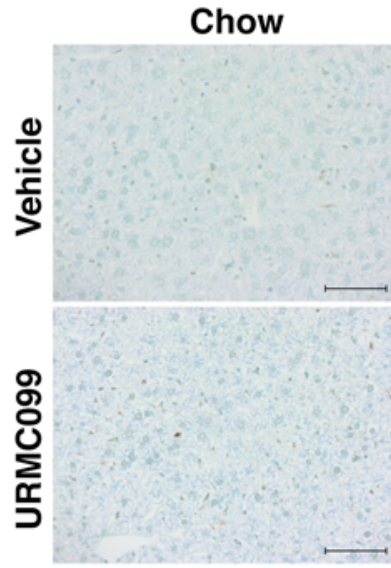

D

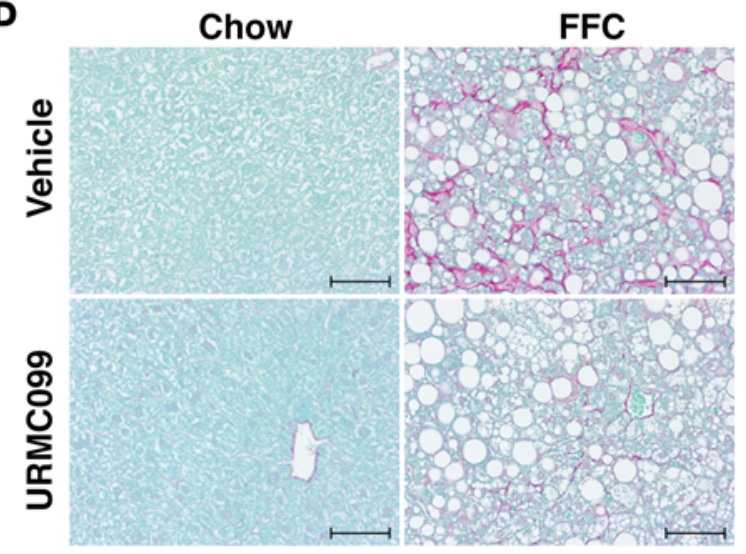

$\mathbf{F}$

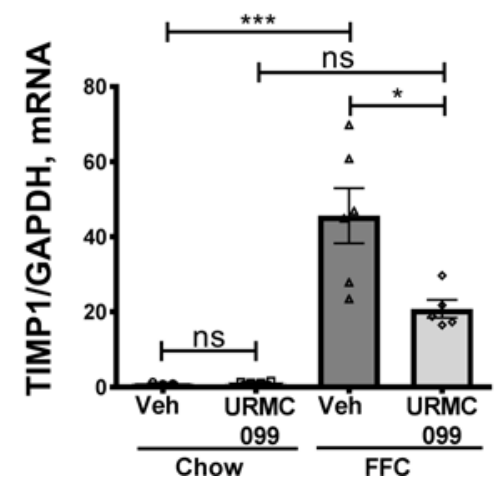

FFC
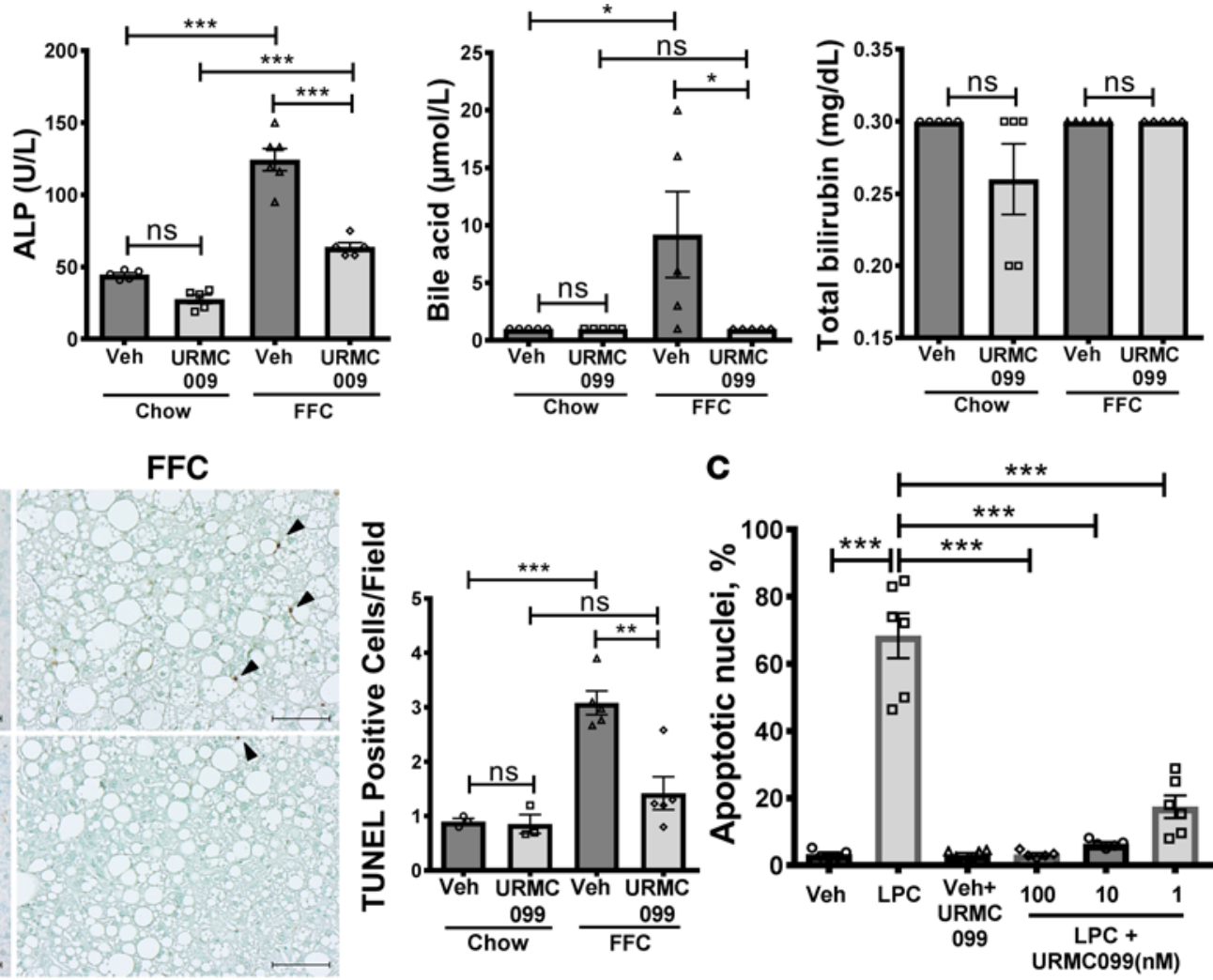

E


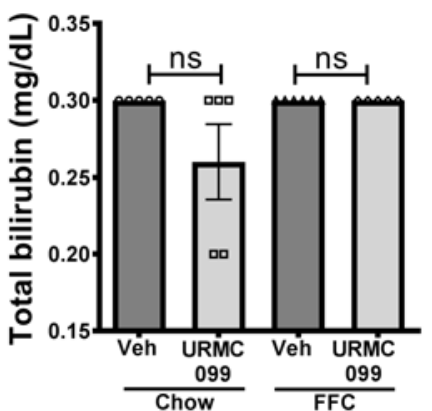

(n)

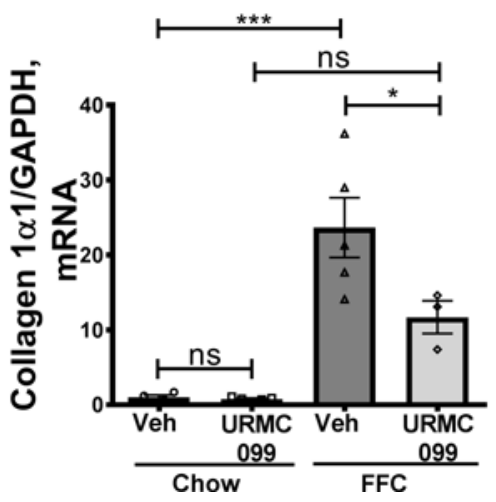

Figure 5. URMC099-treated mice are protected against FFC diet-induced liver injury and fibrosis. WT (57BL/6) mice were fed either chow or a diet high in saturated fat, fructose, and cholesterol (FFC) for 24 weeks, and URMC099 or vehicle (Veh) was given during the last 2 weeks. (A) Serum alanine aminotransferase (ALT), alkaline phosphatase (ALP), bile acid, and total bilirubin were measured ( $n=4-7)$. (B) Hepatocyte apoptosis was assessed by the TUNEL assay. Brown-stained apoptotic nuclei (black arrowheads) were quantified by counting nuclei in 10 random $\times 20$ microscopic fields, and averaged for each animal $(n=3)$. Scale bars: $50 \mu \mathrm{m}$. (C) Huh7 cells were treated with either Veh or $20 \mu \mathrm{M}$ lysophosphatidylcholine (LPC) with or without URMC099 for 16 hours. Apoptosis was assessed by DAPI staining and counting of apoptotic nuclei. (D) Fixed liver tissue sections 
were stained by Sirius red. Sirius red chromogen was quantified by morphometry using Imagel software in 15 random $\times 20$ microscopic fields, and averaged for each animal $(n=3$.) Scale bars: $50 \mu \mathrm{m}$. (E) Frozen liver tissue sections were examined by second harmonic generation (SHG) microscopy using a $\times 25$ objective. Scale bars: $50 \mu \mathrm{m}$. Total RNA was extracted from the liver tissues. The mRNA expression of (F) the profibrogenic markers osteopontin, collagen 1 $\alpha 1$, and TIMP1 were evaluated by real-time PCR $(n=3-5)$. Fold change was determined after normalization to GAPDH mRNA expression, and expressed relative to that observed in chow-fed, vehicle-treated mice. Data represent mean $\pm S E M$. Differences between the groups were compared using 1-way ANOVA followed by Bonferroni's multiple comparisons test. ${ }^{*} P<0.05,{ }^{* *} P<0.01,{ }^{* *} P<0.001$. ns, non-significant.

in vitro. We employed Huh7 cells treated with the toxic lipid lysophosphatidylcholine (LPC), and demonstrated a very potent URMC099-dose-dependent reduction of hepatocyte lipoapoptosis (Figure 5C). We next assessed the protective effect of URMC099 on the FFC diet-induced liver fibrosis by examining Sirius red staining to identify the extent of collagen deposition in liver sections. Sirius red-positive surface area was significantly reduced in FFC-fed, URMC099-treated mice versus vehicle-treated mice (Figure 5D). These findings were confirmed by second harmonic generation (SHG) microscopy for collagen fibrils (Figure 5E). Furthermore, we examined the expression of fibrosis-related genes, including osteopontin, tissue inhibitor of metalloproteinase 1 (TIMP1), and collagen 1a1. The mRNA levels of these genes were increased in the FFC-fed mice, with a significant reduction in the URMC099-treated mice versus vehicletreated mice on the same diet (Figure 5F). Thus, URMC099 appears to be protective against the FFC diet-induced hepatic injury and fibrosis.

URMC099 reduces macrophage activation and migration under lipotoxic conditions. We have demonstrated previously that the pharmacological MLK3 inhibitor URMC099 reduces CXCL10 induction (11), and release by lipotoxic hepatocytes via EVs (23). Furthermore, we have reported that CXCL10-enriched EVs released from lipotoxic hepatocytes induce macrophage chemotaxis (23). In the current study, we sought to advance these observations and explore whether URMC099 has a direct protective effect against macrophage activation and chemotaxis under lipotoxic conditions in vitro. We employed LPC as a proximal mediator of lipotoxicity. LPC is the intracellular metabolite of the SFA palmitate; LPC mimics all the cellular toxicity of palmitate (32-34). We employed lipopolysaccharide (LPS) to simulate the role of gut-derived bacterial products in exacerbating the hepatic inflammation in $\operatorname{NASH}(35,36)$. Since proinflammatory signaling often converges on nuclear factor- $\mathrm{\kappa B}(\mathrm{NF}-\mathrm{\kappa} \mathrm{B})$ to induce transcriptional regulation of inflammatory cytokines such as IL-1 $\beta$ (37), we examined macrophage activation under lipotoxic conditions by assessing the mRNA expression of IL-1 $\beta$ and TNF- $\alpha$ in bone marrow-derived macrophages (BMDMs) treated with LPC and LPS with or without URMC099. Addition of LPS to LPC treatment resulted in an exponential increase in mRNA expression of macrophage activation markers, which were significantly reduced in the presence of URMC099 (Figure 6A). Likewise, URMC099 also reduced LPC-induced activation of Kupffer cells, as assessed by reduced mRNA expression of TNF- $\alpha$ and MCP1 (Figure 6B). To confirm these findings in a monocyte, we employed the human monocyte cell line THP1; URMC099 also reduced LPC-induced activation of THP1, as demonstrated by reduced mRNA expression of TNF- $\alpha$ and MCP1 (Figure 6C). Furthermore, treatment of BMDMs with LPC resulted in NF- $\mathrm{BB}$ pathway activation as evidenced by increased nuclear translocation of p65, an NF- $\mathrm{B}$ component responsible for its transcriptional activity, which was reduced in the presence of the MLK3 inhibitor URMC099 (Figure 6D). We next examined, using a coculture system, whether LPC induces macrophage chemotaxis directly or through its lipotoxic effect on hepatocytes. We employed primary mouse hepatocytes (PMHs) or Huh7 cells that were seeded in a 24-well plate and treated overnight with either vehicle or LPC, with or without $1 \mu \mathrm{M}$ URMC099. The next morning, the treatment media were replaced with serum-free media; hepatocytes were incubated with the serum-free media for an additional 3 hours to allow ample time for potential chemokine secretion by hepatocytes. Fluorescently labeled BMDM or THP-1 cells were seeded on the FluoroBlok inserts creating a coculture system. BMDM or THP-1 cells were allowed to migrate for 4 hours; migrated cells were quantified by the Celigo Imaging Cytometer. We observed a significant increase in macrophage migration toward the hepatocytes that were treated with LPC, which was significantly reduced in the presence of URMC099 (Figure 6E). Interestingly, we did not observe significant migration of macrophages toward the wells that had only LPC-treatment media without hepatocytes (data not shown). Taken together, these observations suggest that URMC099 reduces macrophage activation induced by lipotoxic treatment, bacterial products, or both. Lipotoxic treatment, per se, did not exert a direct chemotactic effect on macrophages, while conditioned media from hepatocytes subjected to lipotoxic treatment induced significant macrophage chemotaxis, which was reduced when hepatocytes were incubated with URMC099 during LPC treatment. These findings are consistent with our previous observation of reduced LPC-induced hepatocyte 


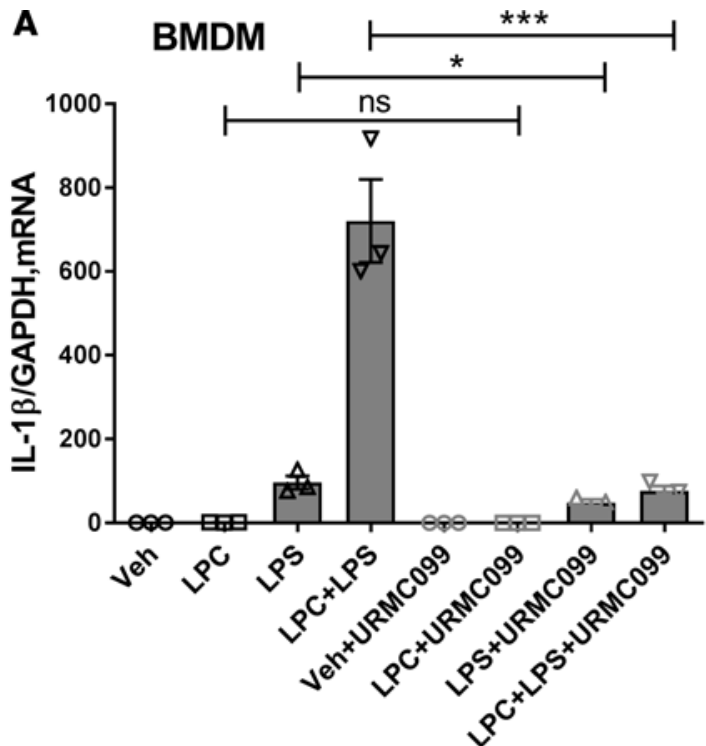

B Kupffer cells

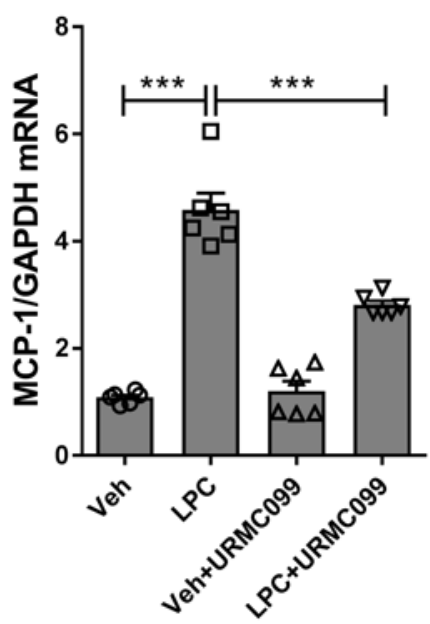

D DAPI





Kupffer cells



Merge

THP-1 cells

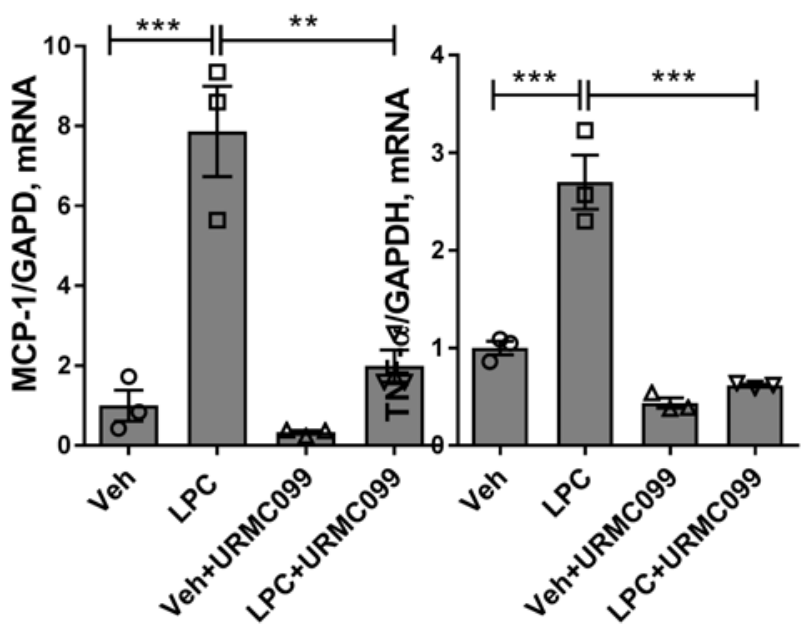

E

BMDM

THP-1 cells
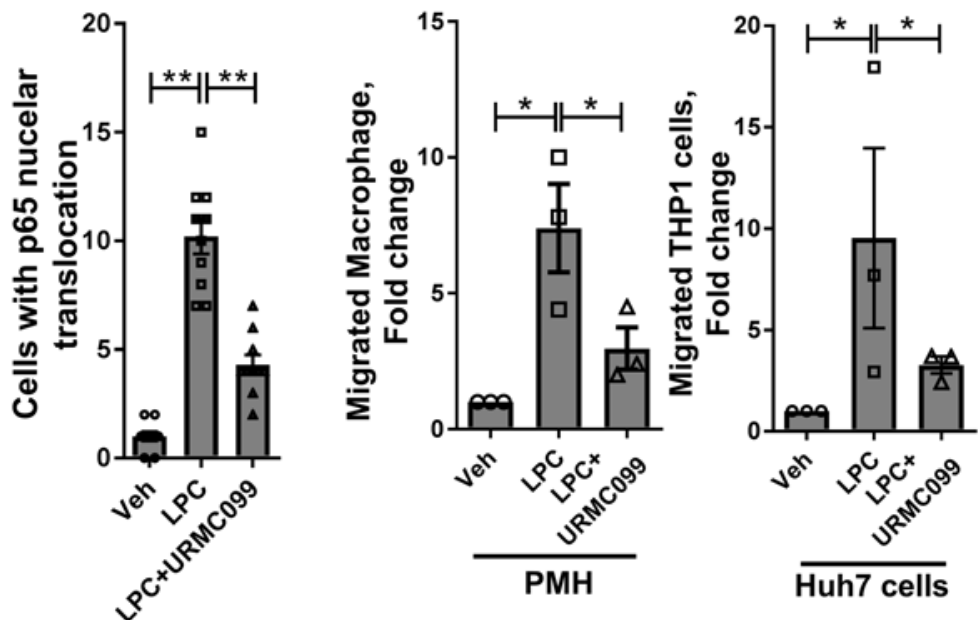

Figure 6. URMC099 reduces macrophage activation and migration under lipotoxic conditions. (A) Bone marrow-derived macrophages (BMDMs) (n = 3) were incubated with either vehicle (Veh), $10 \mu \mathrm{M}$ lysophosphatidylcholine (LPC), or 100 ng/ml lipopolysaccharide (LPS), with or without $100 \mathrm{nM}$ URMC099. (B) Kupffer cells $(n=6-9)$ and $(\mathbf{C})$ THP-1 cells $(n=3)$ were treated with either Veh or LPC (10 $\mu M)$ with or without 100 nM URMC099. After a 4- to 6-hour treatment, total RNA was extracted and mRNA expression for IL-1 $\beta$, TNF- $\alpha$, and MCP1 was assessed by quantitative real-time PCR. Fold change was determined after normalization to GAPDH expression and expressed relative to that observed in vehicle-treated condition. (D) After a 
30-minute $10 \mu \mathrm{M}$ LPC treatment, nuclear localization of p65 in BMDMs was assessed by immunocytochemistry and confocal microscopy. Scale bars: $50 \mu \mathrm{m}$. The p65-positive nuclei were quantified in 10 random microscopic fields. (E) Primary mouse hepatocytes (PMHs) or Huh7 cells were treated overnight with either Veh, LPC, with or without $1 \mu \mathrm{M}$ URMC099, and the treatment media were replaced with serum-free media in the morning.

Fluorescently labeled BMDMs or THP-1 cells were seeded on the cell culture inserts; migrated cells were quantified by the Celigo Imaging Cytometer ( $n$ = 3). Data represent mean \pm SEM. Differences between the groups were compared using 1-way ANOVA followed by Bonferroni's multiple comparisons test. ${ }^{*} P<0.05,{ }^{* *} P<0.01,{ }^{* * *} P<0.001$. ns, non-significant.

CXCL10 expression and release via EVs in the presence of the MLK3 inhibitor URMC099 (23), suggesting that URMC099 attenuates macrophage migration indirectly, partly through suppressing CXCL10-enriched EV release by lipotoxic hepatocytes.

\section{Discussion}

The principal findings of the present study provide both in vivo validation and mechanistic insights regarding the protective effect of the pharmacological MLK3 inhibitor URMC099 in a murine nutritional model of NASH. Our results indicate that (a) URMC099 is well tolerated in FFC-fed mice without any noted adverse effects; (b) URMC099 treatment confers several salutary effects in the reversal of established NASH that are principally mediated by diminished hepatic macrophage trafficking, infiltration, and activation with subsequent decrease in hepatic injury and fibrosis. These observations are independent of obesity and the metabolic changes caused by the FFC diet; (c) URMC099 is protective against macrophage activation, and hepatocyte lipotoxicity-induced macrophage chemotaxis in vitro (Figure 7). To our knowledge, our observations are the first to describe a therapeutic effect of MLK3 pharmacological inhibition in murine NASH. These observations are more thoroughly discussed below.

In this study, we used a well-established dietary model of murine NASH induced by the FFC diet (38, 39). The FFC diet is high in saturated fat, fructose, and cholesterol, and was developed to replicate the Western fast-food diet composition and recapitulate the histological features of human NASH, including neutral lipid accumulation by hepatocytes, ballooned hepatocytes, hepatic inflammatory cell infiltration, and liver fibrosis. The model has a high fidelity to the metabolic profile observed in human NASH, including obesity, hyperlipidemia, and insulin resistance $(24,38)$. In the current study, we demonstrate that the FFC diet induces similar changes in both URMC099 and vehicle-treated mice in the HOMA-IR, body fat composition, metabolic profiles, hepatic steatosis, and TG content analyses.

URMC099-treated mice on the FFC diet have a relative attenuation of all the injurious features of $\mathrm{NASH}$ in this model, without an impact on overall obesity when compared with vehicle-treated mice, including significantly reduced hepatocyte apoptosis, macrophage hepatic infiltration and activation, and hepatic fibrosis compared with vehicle-treated mice on the same diet. Taken together, these observations highlight the key role that the MLK3 signaling pathway plays in the pathogenesis of NASH, and support MLK3 pharmacological inhibition as a therapeutic target with potential disease-modifying outcomes.

We have previously demonstrated that the pharmacological MLK3 inhibitor URMC099 attenuates hepatocyte lipoapoptosis, and sublethal lipotoxic insult manifested by an increase in the EVs released by hepatocytes (23). We have shown that URMC099 suppresses CXCL10 induction in hepatocytes under lipotoxic conditions (11) and its release in CXCL10-enriched EVs in vitro (23). We further demonstrated that these CXCL10-enriched EVs are biologically active and are a very potent inducer of macrophage chemotaxis (23). In the current study, we extend these observations to show that the lipotoxic mediator LPC can activate macrophages, as well as potentiate LPS-induced macrophage activation, an observation consistent with the role of gut-derived bacterial products in exacerbating the hepatic inflammation in NASH (35). Interestingly, we found that URMC099 attenuates macrophage activation induced by both bacterial products and lipotoxic treatment. We further demonstrate, using a coculture system of hepatocytes and macrophages, that URMC099 suppresses macrophage chemotaxis. Exploring the role of URMC099 in suppressing hepatocyte-derived EV release in FFC-fed mice is a subject of our future research. Collectively, these data suggest that the macrophage is also a target for pharmacological MLK3 inhibition in NASH.

In summary, we report a salutary effect of MLK3 pharmacological inhibition with URMC099 in a preclinical model of NASH pathogenesis independent of body weight and the metabolic syndrome. URMC099 appears to be well tolerated in mice without noted adverse effects, consistent with our previous data with longer duration of URMC099 treatment in a different disease model $(25,40)$. Here, we demonstrate that macrophage-associated inflammation is a key player in the MLK3-mediated sterile inflammatory response observed 


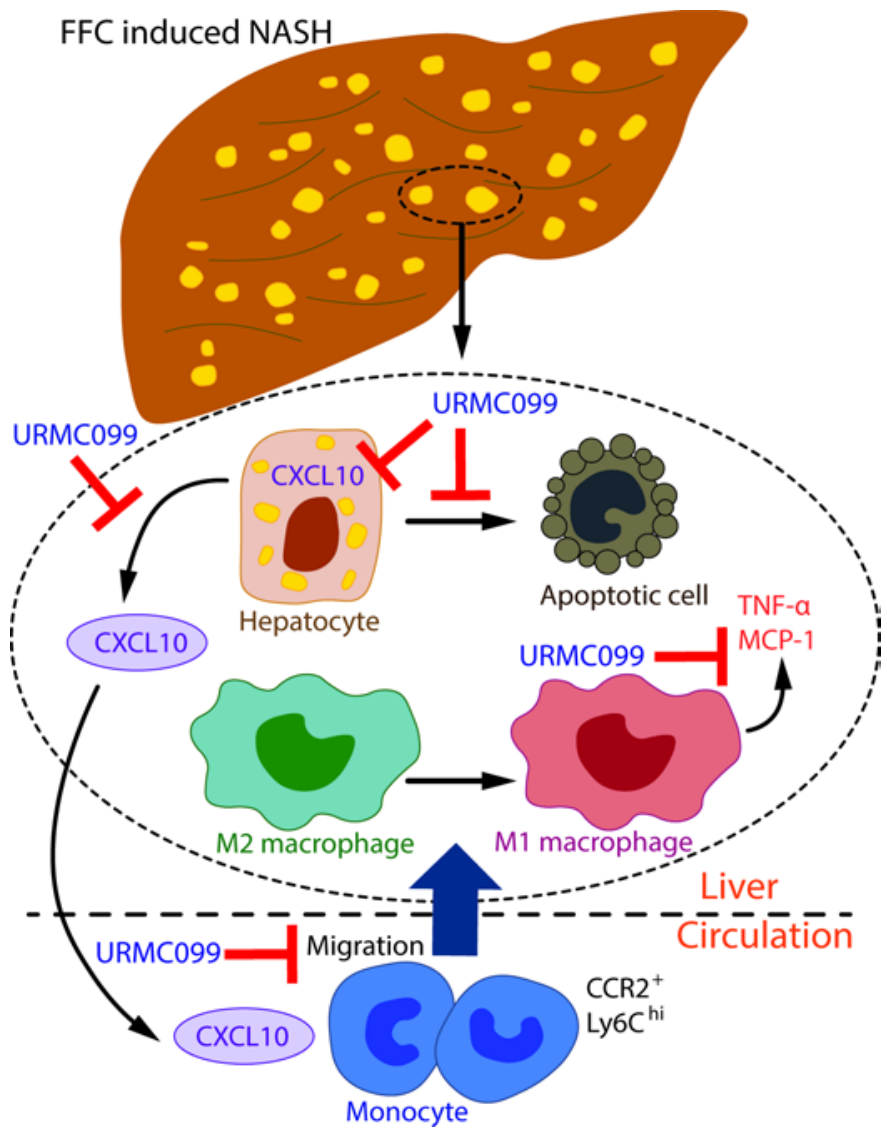

Figure 7. Schematic representation of the protective role of URMC099 against FFC diet-induced murine NASH. URMCO99 is protective against a high-saturated-fat, -fructose, and -cholesterol (FFC) diet-induced hepatocyte apoptosis, CXCL10 induction, and release, resulting in reduced macrophage trafficking to the liver. URMCO99 also reduces macrophage proinflammatory (M1) polarization and activation as assessed by the cytokine (TNF- $\alpha$ ) and chemokine (MCP1) expression. NASH, nonalcoholic steatohepatitis.

in murine NASH. MLK3 inhibition appears to target both the hepatocyte and the macrophage in our murine NASH model. This is not without precedent, since previous reports of URMC099-mediated neuroprotection in models of HIV-1-associated neurocognitive disorders and Alzheimer's disease demonstrate its ability to preserve or restore neurons while reversing the proinflammatory phenotype of the central nervous system-resident professional macrophages (41, 42). URMC099 reduces both lipotoxic lethal and sublethal hepatocyte injury, as well as suppresses both macrophage activation and lipotoxic hepatocyte-induced macrophage chemotaxis in vitro. We have previously developed a congener of URMC099 (URMC-214; US Patents $8,846,909,8,877,772$, and 9,181,247) with a similar profile of antiinflammatory activity that does not penetrate the bloodbrain barrier. We are in the process of devising a large-scale synthetic route for this compound and will begin to characterize its activity in our NASH models in future studies. Therefore, MLK3 inhibition with URMC099 represents a promising therapeutic approach to treat human NASH. We intend to further develop URMC099 for use in early-phase human clinical trials to treat NASH, which remains an important, unmet medical need.

\section{Methods}

Materials. LPC (Sigma-Aldrich) was dissolved as previously described in detail (33) and used at $10 \mu \mathrm{M}$. LPS was obtained from Sigma-Aldrich and used at $50 \mathrm{ng} / \mathrm{ml}$. Primary antisera employed for these studies include p65 (catalog 8242), phospho-MKK3/MKK6 (catalog 12280), phospho-p38 (catalog 9211) from Cell Signaling Technology, and glyceraldehyde 3-phosphate dehydrogenase (GAPDH) (MAB 374) from Millipore. The MLK3 inhibitor URMC099 (25) utilized in the in vitro and in vivo experiments was provided by Harris A. Gelbard (University of Rochester Medical Center, Rochester, New York, USA).

Animals. Eight-week-old male C57BL/6J mice (Jackson Laboratory) on a 12-hour light/dark cycle were used for the experiments. The animals were fed either an FFC diet (40\% calories from fat, $0.2 \%$ cholesterol, AIN-76A Western Diet, TestDiet) (38) plus fructose $23.1 \mathrm{~g} / 1$ and glucose $18.9 \mathrm{~g} / 1$ added to the drinking water, or chow (Teklad-Harlan) diet for 24 weeks. Animals were provided free access to diet for 24 weeks. After 22 weeks on the diet, FFC-fed mice were randomized to either the pharmacological MLK3 inhibitor URMC099 (25) (10 mg/kg) twice daily or its vehicle solution (5\% DMSO, 40\% PEG-400, 55\% normal saline) by i.p. injection for 14 days ( $n=5$ per group). URMC099 was originally designed as a firstin-class MLK3 inhibitor for central nervous system applications and has excellent pharmacokinetic characteristics; specifically, it has a nonlinear shape, few hydrogen bond donors, a favorable Log D (2.45), and a low polar surface area $\left(51 \AA^{2}\right)$ (43). The brain concentration of URMC099 remained well above the in vitro MLK3 IC50 for 6 hours after injection, and the volume of distribution is well within acceptable limits for in vivo use, and suggests URMC099 will not accumulate in tissues. URMC099 is orally bioavailable ( $F$ $=41 \%$ ), but was given by i.p. injection on a twice daily dosing regimen to insure adequate coverage of the target for this experimental paradigm. Unpublished data and data from other studies in different models of systemic and central nervous system HIV-1 infection suggests that once daily dosing (i.p.) may be sufficient to achieve therapeutic effects $(26,40)$. While brain and liver have different cellular and organ architecture, these data suggest that URMC099 is likely to achieve therapeutic levels in organs outside the brain without interference from key human CYP450 enzymes (43). 
Table 1. Mouse primers used for quantitative real-time PCR

\begin{tabular}{|c|c|c|}
\hline Gene & Forward primer sequence $\left(5^{\prime}-3^{\prime}\right)$ & Reverse primer sequence $\left(5^{\prime}-3^{\prime}\right)$ \\
\hline CXCL10 & CCAAGTCCTGCCGTCATTTTC & GGCTCGCAGGGATCATTTCAA \\
\hline CD14 & СTСTCTCCTTAAAGCGGCTTAC & GTTGCGGAGGTTCAAGATGTT \\
\hline $\mathrm{F} 4 / 80$ & CTCTTCTGGGGCTTCAGTGG & GCAGACTGAGTTAGGACCACA \\
\hline Ly6C & GCAGTGCTACGAGTGCTATGG & ACTGACGGGTCTTTAGTTTCCTT \\
\hline CCR2 & ATCCACGGCATACTATCAACATC & CAAGGCTCACCATCATCGTAG \\
\hline IL-1 $1 \beta$ & GCAACTCTTCСTGAАСTСААСТ & ATCTTTTTCGGGTCCGTCAACT \\
\hline IL-12p40 & TGGTTTGCCATCGTTTTGCTG & ACAGGTGAGGTTCACTGTTTCT \\
\hline TIMP1 & AGGTGGTCTCGTTGATTTCT & GTAAGGCCTGTAGCTGTGCC \\
\hline Osteopontin & СTCСАTСGTCATCATCATCG & TGCACCCAGATCCTATAGCC \\
\hline Collagen1a1 & GCTCCTCTTAGGGGCCACT & CCACGTCTCACCATTCGGG \\
\hline
\end{tabular}

During the second week of i.p. injections, metabolic parameters including food intake, oxygen consumption, carbon dioxide production, and locomotor activity were measured using a Comprehensive Laboratory Animal Monitoring System (Columbus Instruments) as previously described (44). Blood glucose was measured using a blood glucose monitor (Assure 4) and plasma insulin was measured by UltraSensitive Mouse Insulin ELISA kit (Crystal Chem. Inc.). HOMA-IR was calculated by using the following formula: HOMA-IR $=26 \times$ fasting insulin level $(\mathrm{ng} / \mathrm{ml}) \times$ fasting glucose level $(\mathrm{mg} / \mathrm{dl}) / 405(45)$. Mice were sacrificed under general anesthesia by ketamine/xylazine cocktail $(83 \mathrm{mg} / \mathrm{kg}$ ketamine $/ 16 \mathrm{mg} / \mathrm{kg}$ xylazine, i.p.) at the end of week 24. Blood and liver samples were collected.

Immunoblot analysis. Whole-liver lysate was obtained using lysis buffer (50 mM Tris- $\mathrm{HCl}, \mathrm{pH} 7.4 ; 1 \%$ Nonidet P-40; 0.25\% sodium deoxycholate; 150 mM NaCl; 1 mM EDTA; 1 mM PMSF; $1 \mu \mathrm{g} / \mathrm{ml}$ aprotinin, leupeptin, pepstatin) followed by centrifugation at $15,000 \mathrm{~g}$ for 15 minutes at $4^{\circ} \mathrm{C}$. Protein concentrations of whole-liver lysates were measured by the Bradford assay method (Sigma-Aldrich). Equal amounts of protein were loaded onto SDS-PAGE gels, transferred to nitrocellulose membranes, and incubated overnight with the primary antibody of interest. Horseradish peroxidase-conjugated secondary antibody (Santa Cruz Biotechnologies) was used at a dilution of a 1:3,000. GAPDH protein levels were used as loading controls. Proteins of interest were detected using enhanced chemiluminescence reagent (Amersham) and Kodak X-OMAT film.

Liver TG, liver enzymes, bilirubin, and bile acid measurement. Liver TG levels were measured in mouse liver homogenates as previously described by Tomita et al. (24). Briefly, $50 \mathrm{mg}$ of wet liver tissue was homogenized in a 5\% NP-40 solution. An EnzyChrom Triglyceride Kit (BioAssay System) was used for the assay according to the manufacturer's instructions. Photometric absorbance was read at $570 \mathrm{~nm}$ using a Synergy H1 microplate reader (BioTek). Serum ALT, alkaline phosphatase, bilirubin, and bile acid were measured by a commercially available veterinary chemistry analyzer (VetScan2, Abaxis Veterinary Diagnostics).

Histology, immunohistochemistry, and digital image analysis. Liver histology was performed using tissue fixed in $10 \%$ formalin, dehydrated, and embedded in paraffin. Sections were stained with H\&E and Sirius red

Table 2. Human primers used for quantitative real-time PCR

\begin{tabular}{|c|c|c|}
\hline Gene & Forward primer sequence $\left(5^{\prime}-3^{\prime}\right)$ & Reverse primer sequence $\left(5^{\prime}-3^{\prime}\right)$ \\
\hline MCP-1 & CAGCCAGATCCAATCAATGCC & TCGAATCCTCAACCCACTTCT \\
\hline
\end{tabular}


stain. TUNEL-positive cells were quantified using the ApopTag Peroxidase in Situ Apoptosis Detection Kit (Millipore), diaminobenzidine (DAB) was used as a peroxidase substrate (Vector Laboratories), and 0.5\% methyl green was used for the counterstain. For immunohistochemistry, formalin-fixed paraffin-embedded liver tissue sections were deparaffinized, hydrated, and stained with antibody against Mac-2 (1:250, eBioscience, catalog 14-5301-82). Bound antibodies were detected using a Vectastain ABC kit (Vector Laboratories) and DAB stain; the tissue sections were counterstained with hematoxylin. Sirius red-stained areas and Mac2-immunopositive areas were quantified by digital image analysis of 10 random fields per slide using the ImageJ software (NIH). Frozen liver sections were imaged on a 2-photon confocal microscope, FluoView FV1000 MPE (Olympus America), using the CARS microscopy application to identify lipid droplets within hepatocytes, and SHG application to identify collagen deposition. Its Mai Tai Deep Sea laser (SpectraPhysics) was tuned to $800 \mathrm{~nm}$ and an XLPlanN 25×/1.05w MP objective lens was used.

Quantitative real-time PCR. Total RNA was isolated with the RNeasy Mini Kit (Qiagen) and was reverse transcribed with moloney murine leukemia virus reverse transcriptase and oligo-dT random primers (both from Invitrogen). Quantification of gene expression was performed by real-time PCR using SYBR green fluorescence on a LightCycler 480 instrument (Roche Applied). Primers are listed in Tables 1 and 2. Target gene expression was calculated using the $\Delta \Delta \mathrm{Ct}$ method and expression was normalized to GAPDH expression levels.

Apoptosis quantification. Huh7 cells were stained with 4',6-diamidino-2-phenylindole (DAPI) for 10 minutes and visualized using a fluorescence microscope. Apoptotic nuclei were quantified as previously described (46).

ELISA. ELISA was performed on sera of vehicle-treated and URMC099-treated mice on chow and FFC diet using the mouse CXCL10 PicoKine ELISA kit (Boster) per the manufacturer's instructions.

Cell lines, primary cell isolation, and culture. The human hepatocellular carcinoma cell line Huh7 was cultured in DMEM containing glucose $(4.5 \mathrm{~g} / 1)$, primocin $(100 \mu \mathrm{g} / \mathrm{ml})$ (InvivoGen), and $10 \%$ fetal bovine serum (FBS, Gibco) under standard conditions. THP-1 cells, a human monocytic cell line, were cultured in RPMI-1640 medium with 2 mM L-glutamine supplemented with 10\% FBS (Gibco). For the activation experiment, THP-1 monocytes were differentiated into macrophages using phorbol 12-myristate 13-acetate ( $5 \mathrm{ng} / \mathrm{ml})$. All animal experiments were performed in accordance with a protocol approved by the Mayo Clinic Institutional Animal Care and Use Committee. PMHs were isolated as previously described (47). In brief, PMHs were isolated from adult mice by collagenase perfusion and purified by Percoll (SigmaAldrich) gradient centrifugation. Up to $5 \times 10^{5}$ isolated hepatocytes were plated onto each well of a collagen-coated 24-well plate. BMDMs were isolated from WT mice as previously described (48, 49). Briefly, bone marrow from dissected mouse tibia and femur was flushed with RPMI-1640 (Gibco) supplemented with $10 \%$ heat-inactivated FBS, $100 \mathrm{U} / \mathrm{ml}$ penicillin, $100 \mu \mathrm{g} / \mathrm{ml}$ streptomycin, and $20 \%$ L929 cell-conditioned media. Cells were resuspended and plated. After 7 days of differentiation, BMDMs were used for subsequent experiments. Kupffer cells were isolated by in situ digestion with pronase and collagenase following cell separation on a discontinuous Percoll gradient and selective adherence (28).

Macrophage chemotaxis. THP-1 and BMDM chemotaxis was assessed by a migration assay using Corning FluoroBlok 24 Well Plate cell culture inserts (product 351152). Huh7 or PMHs were plated in the basolateral chambers the day before, and subjected to the different treatment conditions in $1 \mathrm{ml}$ of serum-free DMEM. After 2 hours of serum starvation, $5 \times 10^{5}$ BMDMs or THP- 1 cells were suspended in $1 \mathrm{ml}$ of RPMI, and then labeled with $5 \mu \mathrm{M}$ Cell Tracker Red CMTPX (Thermo Fisher Scientific) for 45 minutes. Inserts were rehydrated with $500 \mu \mathrm{l}$ of PBS for 2 hours at $37^{\circ} \mathrm{C}$, in an ambient atmosphere incubator. Buffer was removed and $200 \mu 1$ of BMDM or THP-1 suspension was dispensed into the appropriate inserts. The coculture system of either BMDMs and PMHs or THP-1 and Huh7 cells was incubated at $37^{\circ} \mathrm{C}$. BMDMs or THP-1 cells were left to migrate for 4 hours. The migrated cells were imaged and quantified by platebased cytometer Celigo (Nexcelom Bioscience LLC), an automated, kinetic imaging system that assesses and quantifies macrophage migration, using the autofocus feature that allows focusing through the basolateral compartment up to the migrated cell layer on the bottom of the membrane.

Immunofluorescence of $p 65 \mathrm{NF}-\kappa \mathrm{B}$. Immunofluorescence of $\mathrm{p} 65 \mathrm{NF}-\kappa \mathrm{B}$ was performed as previously described by us (49). In brief, BMDMs were serum starved for 2 hours and then incubated with LPC for 30 minutes. Cells were then fixed with $3 \%$ paraformaldehyde, permeabilized with $0.2 \%$ Triton X-100 in PBS, blocked with 5\% BSA in PBS, and the primary antibody p65 (1:400) was applied overnight at $4^{\circ} \mathrm{C}$. Secondary anti-rabbit antibody conjugated to Alexa Fluor 488 (Molecular Probes) was applied for 1 hour. Cells were mounted with ProLong Antifade with DAPI (Molecular Probes) and images were acquired on an LSM780 confocal microscope (Carl Zeiss). Cells with nuclear translocation of p65 NF-kB were quantified. 
Statistics. Data are expressed as the mean \pm SEM. Differences between 2 groups were compared using the Student's $t$ test. Differences between multiple groups were compared using 1-way ANOVA followed by Bonferroni's multiple comparisons test. *, ${ }^{* *}$, and ${ }^{* * *}$, indicate statistical significance with $P<0.05, P<$ 0.01 , and $P<0.001$, respectively. Statistically nonsignificant results were labeled as ns where appropriate. All analyses were performed using GraphPad Prism 6 software.

Study approval. All the study protocols were conducted with approval by the Institutional Animal Care and Use Committee of Mayo Clinic, Rochester, Minnesota, USA.

\section{Author contributions}

KT, BLM, QG, LHGS, PH, and SHI conducted the experiment(s). KT and SHI designed the experiments, analyzed the data, and wrote the manuscript. RK, BCB, and HAG edited the manuscript. All authors reviewed the manuscript.

\section{Acknowledgments}

We thank Gregory J. Gores for his thorough review of the manuscript, Steven F. Bronk for providing excellent technical support, and Courtney N. Hoover for her secretarial support. We also thank Nathan $\mathrm{K}$. LeBrasseur and his laboratory members for their assistance in the Comprehensive Laboratory Animal Monitoring System data. The small-molecule MLK3 inhibitor, URMC099, used in this study is the proprietary asset of the University of Rochester Medical Center (US Patents: 8,846,909, 8,877,772, and 9,181,247, and international patents/applications; References: PMCID: PMC3682381; PMC4032177). This work was supported by the Mayo Clinic Center for Clinical and Translational Science (CCaTS), KL2 program KL2TR000136-09 (to SHI), and pediatric small grant program (to SHI); a Pilot and Feasibility Award and the Optical Microscopy Core of the Mayo Clinic Center for Cell Signaling in Gastroenterology (P30DK084567) (to SHI); North American Society of Pediatric Gastroenterology Hepatology and Nutrition Young Investigator Award/Nestle Nutrition Award (to SHI); Edward C. Kendall Research Fellowship Award (to PH); and the Mayo Clinic, Rochester. Research reported in this publication was supported by the National Institute of Diabetes and Digestive and Kidney Diseases of the NIH under Award Number K08DK111397 (to SHI). The content is solely the responsibility of the authors and does not necessarily represent the official views of the NIH.

Address correspondence to: Samar H. Ibrahim, Pediatric Gastroenterology and Hepatology, Mayo Clinic, 200 First Street SW, Rochester, Minnesota 55905, USA. Phone: 507.284.0686; Email: ibrahim.samar@mayo.edu.

1. Rinella M, Charlton M. The globalization of nonalcoholic fatty liver disease: prevalence and impact on world health. Hepatology. 2016;64(1):19-22.

2. McPherson S, Hardy T, Henderson E, Burt AD, Day CP, Anstee QM. Evidence of NAFLD progression from steatosis to fibrosing-steatohepatitis using paired biopsies: implications for prognosis and clinical management. J Hepatol. 2015;62(5):1148-1155.

3. Angulo P, et al. Liver fibrosis, but no other histologic features, is associated with long-term outcomes of patients with nonalcoholic fatty liver disease. Gastroenterology. 2015;149(2):389-397.e10.

4. Wong RJ, et al. Nonalcoholic steatohepatitis is the second leading etiology of liver disease among adults awaiting liver transplantation in the United States. Gastroenterology. 2015;148(3):547-555.

5. Sanyal AJ, et al. Nonalcoholic steatohepatitis: association of insulin resistance and mitochondrial abnormalities. Gastroenterology. 2001;120(5):1183-1192.

6. Neuschwander-Tetri BA. Hepatic lipotoxicity and the pathogenesis of nonalcoholic steatohepatitis: the central role of nontriglyceride fatty acid metabolites. Hepatology. 2010;52(2):774-788.

7. Hirsova P, Ibrahim SH, Gores GJ, Malhi H. Lipotoxic lethal and sublethal stress signaling in hepatocytes: relevance to NASH pathogenesis. J Lipid Res. 2016;57(10):1758-1770.

8. Puri P, et al. Activation and dysregulation of the unfolded protein response in nonalcoholic fatty liver disease. Gastroenterology. 2008;134(2):568-576.

9. Feldstein AE, et al. Hepatocyte apoptosis and fas expression are prominent features of human nonalcoholic steatohepatitis. Gastroenterology. 2003;125(2):437-443.

10. Brancho D, Ventura JJ, Jaeschke A, Doran B, Flavell RA, Davis RJ. Role of MLK3 in the regulation of mitogen-activated protein kinase signaling cascades. Mol Cell Biol. 2005;25(9):3670-3681.

11. Tomita K, Niibe A, Freeman BL, Bronk SF, Hirsova P, Ibrahim SH. Mixed lineage kinase 3 mediates the induction of CXCL10 by a STAT1-dependent mechanism during hepatocyte lipotoxicity [published online ahead of print March 6, 2017]. J Cell Biochem. doi: 10.1002/jcb.25973.

12. Jaeschke A, Davis RJ. Metabolic stress signaling mediated by mixed-lineage kinases. Mol Cell. 2007;27(3):498-508.

13. Sharma M, Urano F, Jaeschke A. Cdc42 and Rac1 are major contributors to the saturated fatty acid-stimulated JNK pathway in 
hepatocytes. J Hepatol. 2012;56(1):192-198.

14. Humphrey RK, Yu SM, Bellary A, Gonuguntla S, Yebra M, Jhala US. Lysine 63-linked ubiquitination modulates mixed lineage kinase-3 interaction with JIP1 scaffold protein in cytokine-induced pancreatic $\beta$ cell death. J Biol Chem. 2013;288(4):2428-2440.

15. Kim KY, Kim BC, Xu Z, Kim SJ. Mixed lineage kinase 3 (MLK3)-activated p38 MAP kinase mediates transforming growth factor- $\beta$-induced apoptosis in hepatoma cells. J Biol Chem. 2004;279(28):29478-29484.

16. Amako Y, et al. Hepatitis C virus NS5A inhibits mixed lineage kinase 3 to block apoptosis. J Biol Chem. 2013;288(34):24753-24763.

17. Tosello-Trampont AC, Landes SG, Nguyen V, Novobrantseva TI, Hahn YS. Kuppfer cells trigger nonalcoholic steatohepatitis development in diet-induced mouse model through tumor necrosis factor- $\alpha$ production. J Biol Chem. 2012;287(48):40161-40172.

18. Lanthier N. Targeting Kupffer cells in non-alcoholic fatty liver disease/non-alcoholic steatohepatitis: why and how? World $J$ Hepatol. 2015;7(19):2184-2188.

19. Maina V, et al. Bias in macrophage activation pattern influences non-alcoholic steatohepatitis (NASH) in mice. Clin Sci. 2012;122(11):545-553

20. Sica A, Invernizzi P, Mantovani A. Macrophage plasticity and polarization in liver homeostasis and pathology. Hepatology. 2014;59(5):2034-2042.

21. Karlmark KR, et al. Hepatic recruitment of the inflammatory $\mathrm{Gr} 1^{+}$monocyte subset upon liver injury promotes hepatic fibrosis Hepatology. 2009;50(1):261-274.

22. Ibrahim SH, et al. Mixed lineage kinase 3 deficient mice are protected against the high fat high carbohydrate diet-induced steatohepatitis. Liver Int. 2014;34(3):427-437.

23. Ibrahim SH, et al. Mixed lineage kinase 3 mediates release of C-X-C motif ligand 10-bearing chemotactic extracellular vesicles from lipotoxic hepatocytes. Hepatology. 2016;63(3):731-744.

24. Tomita K, et al. CXCL10-mediates macrophage, but not other innate immune cells-associated inflammation in murine nonalcoholic steatohepatitis. Sci Rep. 2016;6:28786.

25. Marker DF, et al. The new small-molecule mixed-lineage kinase 3 inhibitor URMC-099 is neuroprotective and anti-inflammatory in models of human immunodeficiency virus-associated neurocognitive disorders. J Neurosci. 2013;33(24):9998-10010.

26. Dong W, et al. The mixed-lineage kinase 3 inhibitor URMC-099 facilitates microglial amyloid- $\beta$ degradation. J Neuroinflammation. 2016;13(1):184

27. Polesskaya O, et al. MLK3 regulates fMLP-stimulated neutrophil motility. Mol Immunol. 2014;58(2):214-222.

28. Idrissova L, et al. TRAIL receptor deletion in mice suppresses the inflammation of nutrient excess. J Hepatol. 2015;62(5):1156-1163.

29. Khazen W, et al. Expression of macrophage-selective markers in human and rodent adipocytes. FEBS Lett. 2005;579(25):5631-5634

30. Maina V, et al. Bias in macrophage activation pattern influences non-alcoholic steatohepatitis (NASH) in mice. Clin Sci. 2012;122(11):545-553

31. McDonald B, Kubes P. Innate immune cell trafficking and function during sterile inflammation of the liver. Gastroenterology. 2016;151(6):1087-1095

32. Schattenberg JM, Lee MS. Extracellular vesicles as messengers between hepatocytes and macrophages in nonalcoholic steatohepatitis. Gastroenterology. 2016;150(4):815-818

33. Kakisaka K, et al. Mechanisms of lysophosphatidylcholine-induced hepatocyte lipoapoptosis. Am J Physiol Gastrointest Liver Physiol. 2012;302(1):G77-G84.

34. Han MS, et al. Lysophosphatidylcholine as an effector of fatty acid-induced insulin resistance. J Lipid Res. 2011;52(6):1234-1246.

35. Llorente C, Schnabl B. The gut microbiota and liver disease. Cell Mol Gastroenterol Hepatol. 2015;1(3):275-284

36. Abu-Shanab A, Quigley EM. The role of the gut microbiota in nonalcoholic fatty liver disease. Nat Rev Gastroenterol Hepatol. 2010;7(12):691-701

37. Hiscott $\mathrm{J}$, et al. Characterization of a functional NF- $\mathrm{\kappa B}$ site in the human interleukin $1 \beta$ promoter: evidence for a positive autoregulatory loop. Mol Cell Biol. 1993;13(10):6231-6240.

38. Charlton $\mathrm{M}$, et al. Fast food diet mouse: novel small animal model of NASH with ballooning, progressive fibrosis, and high physiological fidelity to the human condition. Am J Physiol Gastrointest Liver Physiol. 2011;301(5):G825-G834.

39. Kim HJ. The physiologic roles of the subepithelial platelet-derived growth factor receptor $\alpha$-positive cells in the colon (Am J Physiol Gastrointest Liver Physiol 2013;304:G823-G834). J Neurogastroenterol Motil. 2014;20(1):127-128.

40. Gnanadhas DP, et al. Autophagy facilitates macrophage depots of sustained-release nanoformulated antiretroviral drugs. $J$ Clin Invest. 2017;127(3):857-873

41. Ma Q, et al. Pharmacokinetic interactions of CEP-1347 and atazanavir in HIV-infected patients. J Neurovirol. 2013;19(3):254-260

42. Dong W, et al. The mixed-lineage kinase 3 inhibitor URMC-099 facilitates microglial amyloid- $\beta$ degradation. J Neuroinflammation. 2016;13(1):184.

43. Goodfellow VS, et al. Discovery, synthesis, and characterization of an orally bioavailable, brain penetrant inhibitor of mixed lineage kinase 3. J Med Chem. 2013;56(20):8032-8048.

44. LeBrasseur NK, Schelhorn TM, Bernardo BL, Cosgrove PG, Loria PM, Brown TA. Myostatin inhibition enhances the effects of exercise on performance and metabolic outcomes in aged mice. J Gerontol A Biol Sci Med Sci. 2009;64(9):940-948

45. Murakami N, et al. Mice heterozygous for the xanthine oxidoreductase gene facilitate lipid accumulation in adipocytes. Arterioscler Thromb Vasc Biol. 2014;34(1):44-51.

46. Cazanave SC, et al. JNK1-dependent PUMA expression contributes to hepatocyte lipoapoptosis. J Biol Chem. 2009;284(39):26591-26602.

47. Hirsova P, Ibrahim SH, Bronk SF, Yagita H, Gores GJ. Vismodegib suppresses TRAIL-mediated liver injury in a mouse mode of nonalcoholic steatohepatitis. PLoS One. 2013;8(7):e70599.

48. Malhi $\mathrm{H}$, et al. C/EBP homologous protein-induced macrophage apoptosis protects mice from steatohepatitis. J Biol Chem 2013;288(26):18624-18642.

49. Hirsova P, et al. Lipid-induced signaling causes release of inflammatory extracellular vesicles from hepatocytes. Gastroenterology. 2016;150(4):956-967. 